\title{
ON TRIPLE SERIES EQUATIONS INVOLVING SERIES OF JACOBI POLYNOMIALS
}

\author{
by K. N. SRIVASTAVA \\ (Received 5th August 1966)
}

\section{Introduction}

Recently Collins (2) has studied triple series equations involving series of Legendre polynomials. These equations arise in the study of mixed boundary value problems and can be regarded as extensions of the dual series equations considered by Collins in (1).

Here we study similar extensions of the dual series equations

$$
\begin{array}{ll}
\sum_{n=0}^{\infty}\left\{A_{n} / \Gamma(n+\alpha+1) \Gamma\left(n+\beta+\frac{3}{2}\right)\right\} P_{n}^{(\alpha, \beta)}(\cos \theta)=f(\theta), & 0 \leqq \theta<a, \\
\sum_{n=0}^{\infty}\left\{A_{n} / \Gamma(n+\beta+1) \Gamma\left(n+\alpha+\frac{1}{2}\right)\right\} P_{n}^{(\alpha, \beta)}(\cos \theta)=g(\theta), & a<\theta \leqq \pi,
\end{array}
$$

where $P_{n}^{(a, \beta)}(\cos \theta)$ is a Jacobi polynomial and $f(\theta)$ and $g(\theta)$ are prescribed. These equations were studied by Srivastav (6). They include the equations considered by Collins (1). The technique employed by Srivastav for solving these equations, however, involves sophisticated assumptions and intricate manipulations. form

In this paper we shall mainly be concerned with triple series equations of the

$$
\begin{array}{ll}
\sum_{n=0}^{\infty}\left\{A_{n} / \Gamma(\beta+n+1) \Gamma\left(n+\alpha+\frac{1}{2}\right)\right\} P_{n}^{(\alpha, \beta)}(\cos \theta)=0, & 0 \leqq \theta<a, \\
\sum_{n=0}^{\infty}\left\{A_{n} / \Gamma(\alpha+n+1) \Gamma\left(n+\beta+\frac{3}{2}\right)\right\} P_{n}^{(\alpha, \beta)}(\cos \theta)=g(\theta), & a<\theta<b, \\
\sum_{n=0}^{\infty}\left\{A_{n} / \Gamma(\beta+n+1) \Gamma\left(n+\alpha+\frac{1}{2}\right)\right\} P_{n}^{(\alpha, \beta)}(\cos \theta)=0, & b<\theta \leqq \pi, \\
& \alpha>-1, \beta>-\frac{1}{2},
\end{array}
$$

defined as triple series equations of the first kind, and those of the form

$$
\begin{array}{ll}
\sum_{n=0}^{\infty}\left\{A_{n} / \Gamma(\alpha+n+1) \Gamma\left(n+\beta+\frac{3}{2}\right)\right\} P_{n}^{(\alpha, \beta)}(\cos \theta)=f(\theta), & 0 \leqq \theta<a, \\
\sum_{n=0}^{\infty}\left\{A_{n} / \Gamma(\beta+n+1) \Gamma\left(n+\alpha+\frac{1}{2}\right)\right\} P_{n}^{(\alpha, \beta)}(\cos \theta)=0, & a<\theta<b, \\
\sum_{n=0}^{\infty}\left\{A_{n} / \Gamma(\alpha+n+1) \Gamma\left(n+\beta+\frac{3}{2}\right)\right\} P_{n}^{(\alpha, \beta)}(\cos \theta)=h(\theta), & b<\theta \leqq \pi, \\
& \alpha>-1, \beta>-\frac{1}{2},
\end{array}
$$


defined as triple series equations of the second kind. The functions $f(\theta), g(\theta)$ and $h(\theta)$ are prescribed and the equations are to be solved for the unknown coefficients $A_{n}$. It is worth noting that when $\alpha=\beta$ we have triple series equations in ultraspherical polynomials. By defining

$$
A_{n}=B_{n} \Gamma(n+\alpha+1) \Gamma\left(n+\alpha+\frac{3}{2}\right)
$$

equations (1.2) to (1.4) have the simple form

$$
\begin{array}{ll}
\sum_{n=0}^{\infty}\left(n+\alpha+\frac{1}{2}\right) B_{n} P_{n}^{(\alpha, \alpha)}(\cos \theta)=0, & (0 \leqq \theta<a, b<\theta \leqq \pi), \\
\sum_{n=0}^{\infty} B_{n} P_{n}^{(\alpha, \alpha)}(\cos \theta)=g(\theta), & a<\theta<b,
\end{array}
$$

with a similar result for equations (1.5) to (1.7). In particular, the cases

(i) $\alpha=\beta=m, m=0,1,2, \ldots$,

(ii) $\alpha=\beta=m+\frac{1}{2}, m=0,1,2, \ldots$

are of special interest, since these triple series equations occur in mixed boundary value problems for spherical rings and strips. When $\alpha=\beta=0$, the equations are discussed by Collins (2) in his study of the electrostatic potential of a spherical ring, while for $\alpha$ equal to a positive integer $m$ the equations occur in the electrostatic potential problem for a spherical ring when the potential is of the form $f(\theta) \cos (m \phi+\alpha)$ on the ring. Since for $\alpha=\frac{1}{2}$

$$
P_{n}^{\left(\frac{1}{2}, \frac{1}{2}\right)}(\cos \theta)=\frac{(2 n+1) !}{2^{2 n}(n) !(n+1) !} U_{n}(\cos \theta),
$$

where $U_{n}(\cos \theta)=\frac{\sin (n+1) \theta}{\sin \theta}$ is a Chebyshev polynomial of the second kind, the equations are then triple series equations in the functions $\sin (n+1) \theta$.

To solve his equations Collins uses a method suggested by work of Gubenko and Massakovskii (5) and replaces the triple series equations by two sets of dual series equations which he solves using the Mehler-Dirichlet integrals for Legendre polynomials. The application of this technique to the series considered here leads to two sets of dual series equations in Jacobi polynomials which cannot be solved by Collins' method. The method used here is therefore different from that of Collins and is similar to one used by Cooke (3) to solve triple integral equations in Bessel functions.

The main result of this paper is that the solution of either set of triple series equations can be reduced to the solution of a Fredholm integral equation of the second kind in one independent variable. It does not appear possible in general to solve this equation exactly, and numerical techniques of solution therefore need to be used. There is a striking similarity between this equation and those obtained by Cooke in his work on triple integral equations.

The analysis given here is purely formal and no attempt is made to justify various limiting processes. 


\section{Some results involving Jacobi polynomials}

It is convenient to list here some results for ready reference. These results are given in (4) and are valid for $\alpha>-1, \beta>-1$. The orthogonality condition for Jacobi polynomials is

$$
\begin{aligned}
\int_{0}^{\pi}\left(\sin \frac{\theta}{2}\right)^{2 \alpha}\left(\cos \frac{\theta}{2}\right)^{2 \beta} P_{n}^{(\alpha, \beta)}(\cos \theta) P_{m}^{(\alpha, \beta)}(\cos \theta) \sin \theta d \theta \\
=\frac{\Gamma(n+\alpha+1) \Gamma(n+\beta+1)}{q_{n}(\alpha, \beta)} \delta_{m n}
\end{aligned}
$$

where $\delta_{m n}$ is a Kronecker delta and $q_{n}(\alpha, \beta)=\frac{1}{2}(n) !(\alpha+\beta+2 n+1) \Gamma(\alpha+\beta+n+1)$. Other results that will be used in later work are the following:

$$
\begin{aligned}
& \int_{0}^{u}\left(\sin \frac{\theta}{2}\right)^{2 \alpha-1}(\cos \theta-\cos u)^{-\frac{1}{2}} P_{n}^{\left(\alpha-\frac{1}{2}, \beta+\frac{1}{2}\right)}(\cos \theta) \sin \theta d \theta \\
& =\frac{(2 \pi)^{\frac{1}{2}} \Gamma\left(n+\alpha+\frac{1}{2}\right)}{\Gamma(n+\alpha+1)}\left(\sin \frac{u}{2}\right)^{2 \alpha} P_{n}^{(\alpha, \beta)}(\cos u) \\
& \int_{u}^{\pi}\left(\cos \frac{\theta}{2}\right)^{2 \beta}(\cos u-\cos \theta)^{-\frac{1}{2}} P_{n}^{(\alpha, \beta)}(\cos \theta) \sin \theta d \theta \\
& =\frac{(2 \pi)^{\frac{1}{2}} \Gamma(n+\beta+1)}{\Gamma\left(n+\beta+\frac{3}{2}\right)}\left(\cos \frac{u}{2}\right)^{2 \beta+1} P_{n}^{\left(\alpha-\frac{1}{2}, \beta+\frac{1}{2}\right)}(\cos u) .
\end{aligned}
$$

We shall use the notation

$$
E(t)=\left(\sin \frac{t}{2}\right)^{2 \alpha}\left(\cos \frac{t}{2}\right)^{-2 \beta} ; K_{a}(u, \theta)=\int_{0}^{a} \frac{E(t) d t}{(\cos t-\cos u)^{\frac{1}{2}}(\cos t-\cos \theta)^{\frac{1}{2}}} .
$$

\section{Variants of Schlomilch's integral equation}

We shall use the following two forms. If $f(\theta)$ and $f^{\prime}(\theta)$ are continuous in $a \leqq \theta \leqq b$, then the solutions of the integral equations

are

$$
\begin{aligned}
& f(\theta)=\int_{a}^{\theta}(\cos u-\cos \theta)^{-\frac{1}{2}} g(u) d u, \\
& f(\theta)=\int_{\theta}^{b}(\cos \theta-\cos u)^{-\frac{1}{2}} g(u) d u,
\end{aligned}
$$

$$
\begin{aligned}
& g(u)=\frac{1}{\pi} \frac{d}{d u} \int_{a}^{u}(\cos \theta-\cos u)^{-\frac{1}{2}} f(\theta) \sin \theta d \theta, \\
& g(u)=-\frac{1}{\pi} \frac{d}{d u} \int_{u}^{b}(\cos u-\cos \theta)^{-\frac{1}{2}} f(\theta) \sin \theta d \theta,
\end{aligned}
$$

respectively. 


\section{A summation result}

Preparatory to the study of the triple series equations, we establish a summation formula. The sum in question is

$$
\begin{aligned}
K(u, \theta) & =\sum_{n=0}^{\infty} \frac{q_{n}(\alpha, \beta) \Gamma\left(n+\alpha+\frac{1}{2}\right)}{\{\Gamma(n+\alpha+1)\}^{2} \Gamma\left(n+\beta+\frac{3}{2}\right)}\left(\sin \frac{u}{2}\right)^{2 \alpha} P_{n}^{(\alpha, \beta)}(\cos u) P_{n}^{(\alpha, \beta)}(\cos \theta) \\
& =\frac{\left(\sin \frac{\theta}{2}\right)^{-2 \alpha}}{\pi} \int_{0}^{\min (u, \theta)} \frac{E(y) d y}{(\cos y-\cos u)^{\frac{3}{2}}(\cos y-\cos \theta)^{\frac{1}{2}}}
\end{aligned}
$$

The result is easily proved. After using (2.2), we have

where

$$
K(u, \theta)=\frac{1}{(2 \pi)^{\frac{1}{2}}} \int_{0}^{u}(\cos y-\cos u)^{-\frac{1}{2}}\left(\sin \frac{y}{2}\right)^{2 \alpha-1} L(y, \theta) \sin \theta d \theta
$$

$$
L(y, \theta)=\sum_{n=0}^{\infty} \frac{q_{n}(\alpha, \beta)}{\Gamma(\alpha+n+1) \Gamma\left(n+\beta+\frac{3}{2}\right)} P_{n}^{\left(\alpha-\frac{1}{2}, \beta+\frac{1}{2}\right)}(\cos y) P_{n}^{(\alpha, \beta)}(\cos \theta) .
$$

With the help of (2.1) and (2.3), it is easy to prove that

$$
L(y, \theta)=\frac{1}{(2 \pi)^{\frac{1}{2}}}\left(\sin \frac{\theta}{2}\right)^{-2 \alpha}\left(\cos \frac{y}{2}\right)^{-2 \beta-1}(\cos y-\cos \theta)^{-\frac{1}{4}} H(\theta-y) .
$$

where $H(t)$ is Heaviside's unit function. Substituting the value of $L(y, \theta)$ from (4.3) in (4.2) we get the required result.

\section{The triple series equations of the first kind}

Let us suppose that for $a<\theta<b$

$$
\sum_{n=0}^{\infty}\left\{A_{n} / \Gamma(n+\beta+1) \Gamma\left(n+\alpha+\frac{1}{2}\right)\right\} P_{n}^{(\alpha, \beta)}(\cos \theta)=n(\theta) .
$$

Using the orthogonality relation (2.1), the above equation along with (1.2) and (1.4), we have

$$
A_{n}=\frac{\Gamma\left(n+\alpha+\frac{1}{2}\right) q_{n}(\alpha, \beta)}{\Gamma(n+\alpha+1)} \int_{a}^{b}\left(\sin \frac{u}{2}\right)^{2 \alpha} P_{n}^{(\alpha, \beta)}(\cos u) n_{1}(u) d u
$$

where $n_{1}(u)=\left(\cos \frac{u}{2}\right)^{2 \beta} \sin u \cdot n(u)$.

If in equation (1.2) we substitute for the coefficients $A_{n}$, we get, on interchanging the order of summation and integration, the equation

$$
g(\theta)=\int_{a}^{b} n_{1}(u) K(u, \theta) d u .
$$

Substituting the value of $K(u, \theta)$ from (4.1), we get

$$
\pi\left(\sin \frac{\theta}{2}\right)^{2 \alpha} g(\theta)=\int_{a}^{b} n_{1}(u) d u \int_{0}^{\min (u, \theta)} \frac{E(y) d y}{(\cos y-\cos \theta)^{\frac{1}{2}}(\cos y-\cos u)^{\frac{1}{2}}} .
$$


We note that the integral in the above equation is

$$
\int_{a}^{b} d u \int_{0}^{\min (u, \theta)} d y=\int_{a}^{\theta} d u \int_{0}^{u} d y+\int_{\theta}^{b} d u \int_{0}^{\theta} d y
$$

and inverting the order of integration we may write this as

$$
\int_{a}^{\theta} d y \int_{y}^{b} d u+\int_{0}^{a} d y \int_{a}^{b} d u
$$

(the integrand being understood).

Hence we have

$$
\begin{aligned}
& \int_{a}^{\theta} \frac{E(y) d y}{(\cos y-\cos \theta)^{\frac{1}{2}}} \int_{y}^{b} \frac{n_{1}(u) d u}{(\cos y-\cos u)^{\frac{1}{2}}} \\
& \quad=\pi\left(\sin \frac{\theta}{2}\right)^{2 \alpha} g(\theta)-\int_{0}^{a} \frac{E(y) d y}{(\cos y-\cos \theta)^{\frac{1}{2}}} \int_{a}^{b} \frac{n_{1}(u) d u}{(\cos y-\cos u)^{\frac{1}{2}}} .
\end{aligned}
$$

We write

$$
N(y)=\int_{y}^{b} \frac{n_{1}(u) d u}{(\cos y-\cos u)^{\frac{1}{2}}}, \quad a<y<b,
$$

and hence from (3.4) we have

$$
n_{1}(u)=-\frac{1}{\pi} \frac{d}{d u} \int_{u}^{b} \frac{N(y) \sin y}{(\cos u-\cos y)^{\frac{1}{2}}} d y .
$$

Equation (5.5) is of the form

From (3.3) we have

$$
\int_{a}^{\theta} \frac{E(y) N(y)}{(\cos y-\cos \theta)^{\frac{1}{2}}} d y=Z(\theta) \text {. }
$$

where

$$
E(y) N(y)=\frac{d}{d y} \int_{a}^{y} \frac{g(\theta)\left(\sin \frac{\theta}{2}\right)^{2 \alpha} \sin \theta}{(\cos \theta-\cos y)^{\frac{1}{2}}} d \theta-\frac{1}{\pi} I, \quad a<y<b,
$$

$$
I=\frac{d}{d y} \int_{a}^{y} \frac{\sin \theta d \theta}{(\cos \theta-\cos y)^{\frac{1}{2}}} \int_{0}^{a} \frac{E(t) d t}{(\cos t-\cos \theta)^{\frac{1}{2}}} \int_{a}^{b} \frac{n_{1}(u) d u}{(\cos t-\cos u)^{\frac{1}{2}}} .
$$

It is easy to prove that

$$
\frac{d}{d y} \int_{0}^{y} \frac{\sin \theta d \theta}{(\cos \theta-\cos y)^{\frac{1}{2}}(\cos t-\cos \theta)^{\frac{1}{2}}}=\frac{\sin y(\cos t-\cos a)^{\frac{1}{2}}}{(\cos a-\cos y)^{\frac{1}{2}}(\cos t-\cos y)} .
$$

Hence

$$
I=\frac{\sin y}{(\cos a-\cos y)^{\frac{1}{2}}} \int_{0}^{a} \frac{E(t)(\cos t-\cos a)^{\frac{1}{2}}}{(\cos t-\cos y)} d t \int_{a}^{b} \frac{n_{1}(u) d u}{(\cos t-\cos u)^{\frac{1}{2}}} .
$$

We write the equation (5.6) in the form

$$
n_{1}(u)=-\frac{1}{\pi} \frac{d J}{d u} \text { where } J=\int_{u}^{b} \frac{N(s) \sin s}{(\cos u-\cos s)^{\frac{1}{2}}} d s
$$


and the last integral in (5.8) in the form

$$
R=-\frac{1}{\pi} \int_{a}^{b} \frac{d J}{d u}(\cos t-\cos u)^{-\frac{1}{2}} d u
$$

On integrating by parts, we get

$$
\begin{aligned}
\pi R= & -\left[\left\{(\cos t-\cos u)^{-\frac{1}{2}} J\right\}\right]_{a}^{b}-\frac{1}{2} \int_{a}^{b} J \frac{\sin u d u}{(\cos t-\cos u)^{\frac{3}{2}}} \\
= & \frac{1}{(\cos t-\cos a)^{\frac{1}{2}}} \int_{a}^{b} \frac{N(s) \sin s d s}{(\cos a-\cos s)^{\frac{1}{2}}} \\
& \quad+\frac{1}{2} \int_{a}^{b} N(s) d s \int_{a}^{s} \frac{\sin u d u}{(\cos t-\cos u)^{\frac{3}{2}}(\cos u-\cos s)^{\frac{1}{2}}}
\end{aligned}
$$

after substituting for $J$ and inverting the order of integration in the last term. Hence

since

$$
R=\frac{1}{\pi(\cos t-\cos a)^{\frac{f}{t}}} \int_{a}^{b} \frac{N(s) \sin s}{(\cos a-\cos s)^{\frac{t}{2}}}\left\{1-\frac{\cos t-\cos a}{\cos t-\cos s}\right\} d s
$$

$$
\int_{a}^{s} \frac{\sin u d u}{(\cos t-\cos u)^{\frac{3}{2}}(\cos u-\cos s)^{\frac{1}{2}}}=\frac{2(\cos a-\cos s)^{\frac{1}{2}}}{(\cos t-\cos s)(\cos t-\cos a)^{\frac{1}{4}}}
$$

Hence

where

$$
I=\frac{1}{\pi} \int_{a}^{b} N(s) B(s, y) d s
$$

$$
\begin{aligned}
& B(s, y)=\frac{\sin s \sin y}{\{(\cos a-\cos y)(\cos a-\cos s)\}^{\frac{1}{2}}} R(s, y), \\
& R(s, y)=\int_{0}^{a} \frac{E(t)(\cos t-\cos a) d t}{(\cos t-\cos s)(\cos t-\cos y)} .
\end{aligned}
$$

Substituting the value of $I$ in (5.7) we get

$$
\begin{aligned}
E(y) N(y)=\frac{d}{d y} \int_{a} \frac{y(\theta)\left(\sin \frac{\theta}{2}\right)^{2 \alpha} \sin \theta}{(\cos \theta-\cos y)^{\frac{1}{2}}} d \theta & \\
& -\frac{1}{\pi^{2}} \int_{a}^{b} N(s) B(s, y) d s, \quad a<y<b .
\end{aligned}
$$

$B(s, y)$ is a symmetric kernel. This is a Fredholm integral equation of the second kind which determines $N(y), n(u)$ being then found from (5.6) and the coefficients $A_{n}$ from (5.2).

In the case $\alpha=\beta, R(s, y)$ can be expressed in terms of hypergeometric unctions. 
We may write for any $\alpha$ and $\beta$

$$
\begin{aligned}
R(s, y)= & \int_{0}^{a}\left(\sin \frac{t}{2}\right)^{2 \alpha}\left(\cos \frac{t}{2}\right)^{-2 \beta} \\
& \left\{\left(\frac{\cos a-\cos s}{\cos y-\cos s}\right) \frac{1}{(\cos t-\cos s)}-\left(\frac{\cos a-\cos y}{\cos y-\cos s}\right) \frac{1}{\cos t-\cos y}\right\} d t,
\end{aligned}
$$

with $a<s, y<b$. Consider therefore

$$
\int_{0}^{a\left(\sin \frac{t}{2}\right)^{2 \alpha}\left(\cos \frac{t}{2}\right)^{-2 \beta}} \quad d t=\left(1+y^{2}\right) \int_{0}^{\tan \frac{a}{2}} \frac{x^{2 \alpha}\left(1+x^{2}\right)^{\beta-\alpha}}{y^{2}-x^{2}} d x
$$

where $y=\tan s / 2$. If $\alpha=\beta$, we can write this integral as

$$
\frac{1+y^{2}}{2 y} \int_{0}^{\tan \frac{a}{2}}\left[\frac{1}{y-x}+\frac{1}{y+x}\right] x^{2 a} d x
$$

and hence we consider the integrals

$$
\begin{aligned}
I & =\int_{0}^{c}(y \pm x)^{-1} x^{2 \alpha} d x, \quad c=\tan \frac{a}{2} \\
& =c^{2 \alpha} v^{-1} \int_{0}^{1} u^{2 \alpha}\left(1 \pm \frac{u}{v}\right)^{-1} d u, \quad y=c v \\
& =\frac{\Gamma(2 \alpha+1)}{\Gamma(2 \alpha+2)} c^{2 \alpha} v^{-1} F_{1}\left[1,2 \alpha+1 ; 2 \alpha+2 ; \pm \frac{1}{v}\right]
\end{aligned}
$$

provided $\alpha>-\frac{1}{2}$. Hence

$R(s, y)=\frac{\left(\tan \frac{a}{2}\right)^{2 \alpha+1}}{(2 \alpha+1)(\cos y-\cos s)}\left[\frac{\cos a-\cos s}{1-\cos s}\{F[z]+F[-z]\}\right.$

where $F[x] \equiv{ }_{2} F_{1}[1,2 \alpha+1 ; 2 \alpha+2 ; x]$

$$
\left.-\frac{\cos a-\cos y}{1-\cos y}\left\{F\left[z_{1}\right]+F\left[-z_{1}\right]\right\}\right]
$$

$$
z=\tan \frac{a}{2} / \tan \frac{s}{2}, \quad z_{1}=\tan \frac{a}{2} / \tan \frac{y}{2}
$$

The hypergeometric functions can be evaluated in terms of elementary functions for certain values of $\alpha$. As pointed out in the introduction the cases

(i) $\alpha=m, m=0,1,2,3, \ldots$,

(ii) $\alpha=m+\frac{1}{2}, m=0,1,2,3, \ldots$,

are of special interest and in these cases $R(s, y)$ can be expressed in terms of 
elementary functions. For example we have

$$
\begin{aligned}
& \alpha=0, R(s, y)=\frac{1}{(\cos y-\cos s)}\left\{\frac{\cos a-\cos s}{\sin s} \log \frac{1+z}{1-z}\right. \\
& \left.-\frac{\cos a-\cos y}{\sin y} \log \frac{1+z_{1}}{1-z_{1}}\right\} \\
& \alpha=\frac{1}{2}, R(s, y)=\frac{1}{2(\cos y-\cos s)}\left\{\frac{\cos a-\cos y}{\cos ^{2} \frac{y}{2}} \log \left(1-z_{1}^{2}\right)\right. \\
& \left.-\frac{\cos a-\cos s}{\cos ^{2} \frac{s}{2}} \log \left(1-z^{2}\right)\right\} \\
& \alpha=1, R(s, y)=\frac{1}{2(\cos y-\cos s)}\left\{\frac{\sin \frac{s}{2}(\cos a-\cos s)}{\cos ^{3} \frac{s}{2}}\left(\log \frac{1+z}{1-z}-z\right)\right. \\
& \left.-\frac{\sin \frac{y}{2}(\cos a-\cos y)}{\cos ^{3} \frac{y}{2}}\left(\log \frac{1+z_{1}}{1-z_{1}}-z_{1}\right)\right\} .
\end{aligned}
$$

\section{Triple series equations of the second kind}

We begin with the assumption

$$
\begin{array}{ll}
\sum_{n=0}^{\infty}\left\{A_{n} / \Gamma(n+\beta+1) \Gamma\left(n+\alpha+\frac{1}{2}\right)\right\} P_{n}^{(\alpha, \beta)}(\cos \theta)=l(\theta), & 0 \leqq \theta<a, \\
\sum_{n=0}^{\infty}\left\{A_{n} / \Gamma(n+\beta+1) \Gamma\left(n+\alpha+\frac{1}{2}\right)\right\} P_{n}^{(\alpha, \beta)}(\cos \theta)=m(\theta), & b<\theta \leqq \pi .
\end{array}
$$

The above equation, the equation (1.6) and the orthogonality relation (2.1) give $A_{n}=\frac{q_{n}(\alpha, \beta) \Gamma\left(n+\alpha+\frac{1}{2}\right)}{\Gamma(n+\alpha+1)}\left\{\int_{0}^{a} l_{1}(u)+\int_{b}^{\pi} m_{1}(u)\right\}\left\{\sin \frac{u}{2}\right\}^{2 \alpha} P_{n}^{(\alpha, \beta)}(\cos u) d u$

where

$$
l_{1}(u)=\left(\cos \frac{u}{2}\right)^{2 \beta} \sin u \cdot l(u), \quad m_{1}(u)=\left(\cos \frac{u}{2}\right)^{2 \beta} \sin u \cdot m(u) .
$$

Substituting for the coefficients $A_{n}$ from (6.3) in the equations (1.5) and (1.7), we find on interchanging the order of summation and integration that

$$
\begin{aligned}
& f(\theta)=\left\{\int_{0}^{a} l_{1}(u)+\int_{b}^{\pi} m_{1}(u)\right\} K(u, \theta) d u, \quad 0 \leqq \theta<a, \\
& h(\theta)=\left\{\int_{0}^{a} l_{1}(u)+\int_{b}^{\pi} m_{1}(u)\right\} K(u, \theta) d u, \quad b<\theta \leqq \pi .
\end{aligned}
$$


TRIPLE SERIES EQUATIONS OF JACOBI POLYNOMIALS 229 where $K(u, \theta)$ is defined by equation (4.1).

We deal with (6.4) in the same way as in $\S 5$. This gives

where

$$
\int_{y}^{a} \frac{l_{1}(u) d u}{(\cos y-\cos u)^{\frac{1}{2}}}=F(y)-\int_{b}^{\pi} \frac{m_{1}(u) d u}{(\cos y-\cos u)^{\frac{t}{2}}}, \quad 0 \leqq y<a,
$$

Using (3.4) we get

$$
F(y)=\frac{1}{E(y)} \frac{d}{d y} \int_{0}^{y} \frac{\left(\sin \frac{\theta}{2}\right)^{2 \alpha} \sin \theta f(\theta)}{(\cos \theta-\cos y)^{\frac{1}{2}}} d \theta .
$$

$l_{1}(u)=F_{1}(u)-\frac{1}{\pi} \frac{\sin u}{(\cos u-\cos a)^{\frac{1}{2}}} \int_{b}^{\pi} \frac{m_{1}(t)(\cos a-\cos t)^{\frac{1}{2}}}{(\cos u-\cos t)} d t, \quad 0 \leqq u<a$,

where

$$
F_{1}(u)=-\frac{1}{\pi} \frac{d}{d u} \int_{u}^{a} \frac{F(y) \sin y}{(\cos u-\cos y)^{\frac{7}{4}}} d u .
$$

We next consider the equation (6.5) which can be written as

$$
\begin{aligned}
\pi\left(\sin \frac{\theta}{2}\right)^{2 \alpha} h(\theta)=\int_{0}^{a} l_{1}(u) K_{u}(u, \theta) d u+ & \int_{b}^{\pi} m_{1}(u) d u \\
& \int_{0}^{\min (u, \theta)} \frac{E(y) d y}{(\cos y-\cos u)^{\frac{1}{2}}(\cos y-\cos \theta)^{\frac{1}{2}}} .
\end{aligned}
$$

We deal with the last integral as in $\S 5$. Hence we get

$$
\begin{aligned}
\int_{b}^{\theta} \frac{E(y) M(y) d y}{(\cos y-\cos \theta)^{\frac{1}{2}}}=\pi\left(\sin \frac{\theta}{2}\right)^{2 \alpha} h(\theta)-\int_{0}^{a} l_{1}(u) K_{u}(u, \theta) d u \\
\\
-\int_{b}^{\pi} m_{1}(u) K_{b}(u, \theta) d u, \quad b<\theta \leqq \pi
\end{aligned}
$$

where

From (3.4) we have

$$
M(y)=\int_{y}^{\pi} \frac{m_{1}(u) d u}{(\cos y-\cos u)^{\frac{1}{x}}}
$$

Hence

$$
m_{1}(u)=-\frac{1}{\pi} \frac{d}{d u} \int_{u}^{\pi} \frac{M(y) \sin y d y}{(\cos u-\cos y)^{\frac{1}{2}}} .
$$

$$
E(y) M(y)=\frac{d}{d y} \int_{b}^{y} \frac{\left(\sin \frac{\theta}{2}\right)^{2 \alpha} h(\theta) \sin \theta}{(\cos \theta-\cos y)^{\frac{1}{2}}} d \theta-\frac{1}{\pi}\left(A_{1}+A_{2}\right)
$$

where

$$
\begin{aligned}
& A_{1}=\frac{d}{d y} \int_{b}^{y} \frac{\sin \theta d \theta}{(\cos \theta-\cos y)^{\frac{1}{2}}} \int_{0}^{a} l_{1}(u) K_{u}(u, \theta) d u, \\
& A_{2}=\frac{d}{d y} \int_{b}^{y} \frac{\sin \theta d \theta}{(\cos \theta-\cos y)^{\frac{1}{t}}} \int_{b}^{\pi} m_{1}(u) K_{b}(u, \theta) d u .
\end{aligned}
$$


After some manipulations we can write

$$
A_{1}=\frac{\sin y}{(\cos b-\cos y)^{\frac{1}{2}}} \int_{0}^{a} \frac{E(t)(\cos t-\cos b)^{\frac{1}{2}} d t}{(\cos t-\cos y)} \int_{t}^{a} \frac{l_{1}(u)}{(\cos t-\cos u)^{\frac{1}{2}}} d u .
$$

Introducing the value of the last integral in the above equation from (6.6) we get

$$
\frac{(\cos b-\cos y)^{\frac{1}{2}}}{\sin y} A_{1}=F_{2}(y)-\int_{b}^{\pi} m_{1}(u) d u \int_{0}^{a} \frac{E(t)(\cos t-\cos b)^{\frac{1}{2}} d t}{(\cos t-\cos y)(\cos t-\cos u)^{\frac{1}{2}}}
$$

where

$$
F_{2}(y)=\int_{0}^{a} \frac{E(t) F(t)(\cos t-\cos b)^{\frac{1}{2}}}{(\cos t-\cos y)} d t .
$$

Similarly $A_{2}$ can be written as

$$
A_{2}=\int_{b}^{\pi} m_{1}(u) d u \int_{0}^{b} \frac{E(t)(\cos t-\cos b)^{\frac{1}{2}} \sin y}{\{(\cos t-\cos u)(\cos b-\cos y)\}^{\frac{1}{2}}(\cos t-\cos y)} d t .
$$

Hence

where

$$
\frac{(\cos b-\cos y)^{\frac{1}{2}}\left(A_{1}+A_{2}\right)}{\sin y}=F_{2}(y)+\int_{a}^{b} \frac{E(t)(\cos t-\cos b)^{\frac{1}{2}}}{(\cos t-\cos y)} R_{1} d t
$$

$$
R_{1}=\int_{b}^{\pi} \frac{m_{1}(u) d u}{(\cos t-\cos u)^{\frac{3}{2}}}=-\frac{1}{\pi} \int_{b}^{\pi} \frac{d J_{1}}{d u(\cos } \frac{d u}{t-\cos u)^{\frac{1}{2}}}
$$

since from (6.9) we have

$$
J_{1}=\int_{u}^{\pi} \frac{M(s) \sin s}{(\cos u-\cos s)^{\frac{1}{2}}} d s .
$$

Integrating by parts, we get

$$
\pi R_{1}=\frac{1}{(\cos t-\cos b)^{\frac{1}{2}}} \int_{b}^{\pi} \frac{M(s)(\cos t-\cos b) \sin s}{(\cos b-\cos s)^{\frac{1}{2}}(\cos t-\cos s)} d s .
$$

The equation (6.10) can be written as

$$
\begin{array}{r}
E(y) M(y)=\frac{d}{d y} \int_{b} \frac{\left(\sin \frac{\theta}{2}\right)^{2 \alpha} \sin \theta h(\theta)}{(\cos \theta-\cos y)^{\frac{1}{2}}} d \theta-\frac{F_{2}(y) \sin y}{\pi(\cos b-\cos y)^{\frac{1}{2}}} \\
-\frac{1}{\pi^{2}} \int_{b}^{\pi} M(s) T(s, y) d s, \quad b<y \leqq \pi
\end{array}
$$

where

$$
T(s, y)=\frac{\sin y \sin s}{\{(\cos b-\cos y)(\cos b-\cos s)\}^{\frac{t}{2}}} \int_{a}^{b} \frac{E(t)(\cos t-\cos b) d t}{(\cos t-\cos s)(\cos t-\cos y)}
$$

is a symmetric kernel. In (6.11) the first two terms on the right-hand side are known functions. This is a Fredholm integral equation of the second kind which determines $M(y), m_{1}(u)$ being then calculated from (6.9). Then $l_{1}(u)$ is 
TRIPLE SERIES EQUATIONS OF JACOBI POLYNOMIALS 231 determined from (6.7) and the coefficients $A_{n}$ are calculated from the equation (6.3).

I am grateful to the referee for his suggestions.

\section{REFERENCES}

(1) W. D. Colunns, Proc. Camb. Phil. Soc. 57 (1961), 367-384.

(2) W. D. Coluins, Archive Rational Mech. App. Math. 16 (1962), 122-137.

(3) J. C. CoOKe, Quart. J. App. Math. 16 (1963), 193-203.

(4) A. ERDelyi, Tables of Integral Transforms, vol. 2 (McGraw-Hill, 1954).

(5) V. S. Gubenko and V. I. MossakovsKI, P.M.M. 24 (1960), 334-340.

(6) R. P. Srivastav, Proc. Roy. Soc. Edin. A, 66 (1964), 185-191.

M. A. College of Technology

BHOPAL (M. P.) INDIA 